\title{
Existence of Nonoscillatory Solutions for a Third-Order Nonlinear Neutral Delay Differential Equation
}

\author{
Zeqing Liu, ${ }^{1}$ Lin Chen, ${ }^{1}$ Shin Min Kang, ${ }^{2}$ and Sun Young $\mathrm{Cho}^{3}$ \\ ${ }^{1}$ Department of Mathematics, Liaoning Normal University, Dalian, Liaoning 116029, China \\ ${ }^{2}$ Department of Mathematics and RINS, Gyeongsang National University, \\ Jinju 660-701, Republic of Korea \\ ${ }^{3}$ Department of Mathematics, Gyeongsang National University, Jinju 660-701, Republic of Korea \\ Correspondence should be addressed to Shin Min Kang, smkang@gnu.ac.kr
}

Received 4 April 2011; Accepted 28 June 2011

Academic Editor: Elena Braverman

Copyright (c) 2011 Zeqing Liu et al. This is an open access article distributed under the Creative Commons Attribution License, which permits unrestricted use, distribution, and reproduction in any medium, provided the original work is properly cited.

The aim of this paper is to study the solvability of a third-order nonlinear neutral delay differential equation of the form $\left\{\alpha(t)\left[\beta(t)(x(t)+p(t) x(t-\tau))^{\prime}\right]^{\prime}\right\}^{\prime}+f\left(t, x\left(\sigma_{1}(t)\right), x\left(\sigma_{2}(t)\right), \ldots, x\left(\sigma_{n}(t)\right)\right)=0$, $t \geq t_{0}$. By using the Krasnoselskii's fixed point theorem and the Schauder's fixed point theorem, we demonstrate the existence of uncountably many bounded nonoscillatory solutions for the above differential equation. Several nontrivial examples are given to illustrate our results.

\section{Introduction and Preliminaries}

In recent years, the study of the oscillation, nonoscillation, asymptotic behaviors and existence of solutions for various kinds of first- and second-order neutral delay differential equations and systems of differential equations have attracted much attention, for example, see [1-12] and the references therein. Dorociaková and Olach [2] discussed the existence of nonoscillatory solutions and asymptotic behaviors for the first-order delay differential equation

$$
x^{\prime}(t)+p(t) x(t)+q(t) x(\tau(t))=0, \quad t \geq 0 .
$$


Elbert [3] and Huang [5] established a few oscillation and nonoscillation criteria for the second-order linear differential equation

$$
x^{\prime \prime}(t)+q(t) x(t)=0, \quad t \geq 0,
$$

where $q \in C\left([0,+\infty), \mathbb{R}^{+}\right)$. Tang and Liu [10] studied the existence of bounded oscillation for the second-order linear delay differential equation of unstable type

$$
x^{\prime \prime}(t)=p(t) x(t-\tau), \quad t \geq t_{0},
$$

where $\tau>0, p \in C\left(\left[t_{0},+\infty\right), \mathbb{R}^{+}\right)$and $p(t) \not \equiv 0$ on any interval of length $\tau$. In view of the Banach fixed point theorem, Kulenović and Hadžiomerspahić [7] deduced the existence of a nonoscillatory solution for the second-order linear neutral delay differential equation with positive and negative coefficients

$$
[x(t)+c x(t-\tau)]^{\prime \prime}+Q_{1}(t) x\left(t-\sigma_{1}\right)-Q_{2}(t) x\left(t-\sigma_{2}\right)=0, \quad t \geq t_{0},
$$

where $c \in \mathbb{R} \backslash\{-1,1\}, \tau>0, \sigma_{1}, \sigma_{2} \in[0,+\infty), Q_{1}, Q_{2} \in C\left(\left[t_{0},+\infty\right), \mathbb{R}^{+}\right)$. Qin et al. [9] and Yang et al. [11] developed several oscillation criteria for the second-order differential equation

$$
\left[r(t)(x(t)+p(t) x(t-\tau))^{\prime}\right]^{\prime}+q(t) f(x(t-\delta))=0, \quad t \geq t_{0}
$$

where $\tau$ and $\delta$ are nonnegative constants, $r, p, q \in C\left(\left[t_{0},+\infty\right), \mathbb{R}\right)$, and $f \in C(\mathbb{R}, \mathbb{R})$. By utilizing the Krasnoselskii's fixed point theorem, Zhou [12] discussed the existence of nonoscillatory solutions of the second-order nonlinear neutral differential equation

$$
\left[r(t)(x(t)+p(t) x(t-\tau))^{\prime}\right]^{\prime}+\sum_{i=1}^{m} Q_{i}(t) f\left(x_{i}\left(t-\sigma_{i}\right)\right)=0, \quad t \geq t_{0}
$$

where $m \geq 1$ is an integer, $\tau>0, \sigma_{i} \geq 0, r, p, Q_{i} \in C\left(\left[t_{0},+\infty\right), \mathbb{R}\right)$, and $f_{i} \in C(\mathbb{R}, \mathbb{R})$ for $i \in\{1,2, \ldots, m\}$.

However, the existence of nonoscillatory solutions of third-order neutral differential equations received much less attention, moreover, the results in $[7,11,12]$ only figured out the existence of a nonoscillatory solution of (1.3)-(1.5), respectively.

Motivated by the papers mentioned above, in this paper, we investigate the following third-order nonlinear neutral delay differential equation

$$
\left\{\alpha(t)\left[\beta(t)(x(t)+p(t) x(t-\tau))^{\prime}\right]^{\prime}\right\}^{\prime}+f\left(t, x\left(\sigma_{1}(t)\right), x\left(\sigma_{2}(t)\right), \ldots, x\left(\sigma_{n}(t)\right)\right)=0, \quad t \geq t_{0},
$$

where $n \geq 1$ is an integer, $\tau>0, \alpha, \beta \in C\left(\left[t_{0},+\infty\right), \mathbb{R}^{+} \backslash\{0\}\right), p \in C\left(\left[t_{0},+\infty\right), \mathbb{R}\right)$, and $f \in$ $C\left(\left[t_{0},+\infty\right) \times \mathbb{R}^{n}, \mathbb{R}\right)$. By applying the Krasnoselskii's fixed point theorem and the Schauder's fixed point theorem, we obtain some sufficient conditions for the existence of uncountably many bounded nonoscillatory solutions of (1.7). 
Throughout this paper, we assume that $\mathbb{R}=(-\infty,+\infty), \mathbb{R}^{+}=[0,+\infty), C\left(\left[t_{0},+\infty\right), \mathbb{R}\right)$ denotes the Banach space of all continuous and bounded functions on $\left[t_{0},+\infty\right)$ with the norm $\|x\|=\sup _{t \geq t_{0}}|x(t)|$ for each $x \in C\left(\left[t_{0},+\infty\right), \mathbb{R}\right)$ and

$$
A(N, M)=\left\{x \in C\left(\left[t_{0},+\infty\right), \mathbb{R}\right): N \leq x(t) \leq M, t \geq t_{0}\right\} \quad \text { for } M>N>0
$$

It is easy to see that $A(N, M)$ is a bounded closed and convex subset of $C\left(\left[t_{0},+\infty\right), \mathbb{R}\right)$.

By a solution of (1.7), we mean a function $x \in C\left(\left[t_{1}-\tau,+\infty\right), \mathbb{R}\right)$ for some $t_{1} \geq t_{0}$ such that $x(t)+p(t) x(t-\tau), \beta(t)(x(t)+p(t) x(t-\tau))^{\prime}$ and $\alpha(t)\left[\beta(t)(x(t)+p(t) x(t-\tau))^{\prime}\right]^{\prime}$ are continuously differentiable in $\left[t_{1},+\infty\right)$ and such that (1.7) is satisfied for $t \geq t_{1}$. As is customary, a solution of (1.7) is called oscillatory if it has arbitrarily large zeros, and otherwise it is said to be nonoscillatory.

Definition 1.1 (see [6]). A family $F$ of functions in $C\left(\left[t_{0},+\infty\right), \mathbb{R}\right)$ is equicontinuous on $\left[t_{0},+\infty\right)$ if for any $\varepsilon>0$, the interval $\left[t_{0},+\infty\right)$ can be decomposed into a finite number of subintervals $I_{1}, I_{2}, \ldots, I_{n}$ such that

$$
|f(x)-f(y)| \leq \varepsilon, \quad \forall f \in F, x, y \in I_{i}, i \in\{1,2, \ldots, n\} .
$$

Lemma 1.2 (see Krasnoselskii's fixed point theorem, [4]). Let $X$ be a Banach space. Let $\Omega$ be a bounded closed convex subset of X and $S_{1}$ and $S_{2}$ mappings from $\Omega$ into X such that $S_{1} x+S_{2} y \in \Omega$ for every pair $x, y \in \Omega$. If $S_{1}$ is a contraction and $S_{2}$ is completely continuous, then the equation $S_{1} x+S_{2} x=x$ has at least one solution in $\Omega$.

Lemma 1.3 (see Schauder's fixed point theorem, [4]). Let $\Omega$ be a nonempty closed convex subset of a Banach space X. Let $S: \Omega \rightarrow \Omega$ be a continuous mapping such that $S \Omega$ is a relatively compact subset of X. Then $S$ has at least one fixed point in $\Omega$.

\section{Main Results}

Now we study those conditions under which (1.7) possesses uncountably many bounded nonoscillatory solutions.

Theorem 2.1. Assume that there exist constants $M, N, c_{1}, c_{2}, T_{0}$ and a function $h \in C\left(\left[t_{0},+\infty\right), \mathbb{R}^{+}\right)$ satisfying

$$
\begin{gathered}
\min \left\{c_{1}, c_{2}\right\} \geq 0, \quad c_{1}+c_{2}<1, \quad 0<N<\left(1-c_{1}-c_{2}\right) M, \\
-c_{2} \leq p(t) \leq c_{1}, \quad t \geq T_{0}>t_{0} ; \\
\left|f\left(t, u_{1}, u_{2}, \ldots, u_{n}\right)\right| \leq h(t), \quad t \in\left[t_{0},+\infty\right), \quad u_{i} \in[N, M], \quad i \in\{1, \ldots, n\} ; \\
\int_{t_{0}}^{+\infty} \int_{v}^{+\infty} \int_{s}^{+\infty} \frac{h(u)}{\alpha(s) \beta(v)} d u d s d v<+\infty .
\end{gathered}
$$

Then (1.7) possesses uncountably many bounded nonoscillatory solutions in $A(N, M)$. 
Proof. Set $k \in\left(c_{1} M+N,\left(1-c_{2}\right) M\right)$. From (2.4), we pick up $T>T_{0}$ such that

$$
\int_{T}^{+\infty} \int_{v}^{+\infty} \int_{s}^{+\infty} \frac{h(u)}{\alpha(s) \beta(v)} d u d s d v<\min \left\{\left(1-c_{2}\right) M-k, k-c_{1} M-N\right\}
$$

Define two mappings $S_{1 k}$ and $S_{2 k}: A(N, M) \rightarrow C\left(\left[t_{0},+\infty\right), \mathbb{R}\right)$ by

$$
\begin{gathered}
\left(S_{1 k} x\right)(t)= \begin{cases}k-p(t) x(t-\tau), & t \geq T, \\
\left(S_{1 k} x\right)(T), & t_{0} \leq t<T,\end{cases} \\
\left(S_{2 k} x\right)(t)= \begin{cases}\int_{t}^{+\infty} \int_{v}^{+\infty} \int_{s}^{+\infty} \frac{1}{\alpha(s) \beta(v)} f\left(u, x\left(\sigma_{1}(u)\right), \ldots, x\left(\sigma_{n}(u)\right)\right) d u d s d v, & t \geq T, \\
\left(S_{2 k} x\right)(T), & t_{0} \leq t<T\end{cases}
\end{gathered}
$$

for $x \in A(N, M)$.

Firstly, we prove that $S_{1 k}(A(N, M))+S_{2 k}(A(N, M)) \subseteq A(N, M)$. By (2.1)-(2.3) and (2.5)-(2.7), we get that

$$
\begin{aligned}
\left(S_{1 k} x\right)(t)+\left(S_{2 k} y\right)(t) & \leq k+c_{2} M+\int_{T}^{+\infty} \int_{v}^{+\infty} \int_{s}^{+\infty} \frac{h(u)}{\alpha(s) \beta(v)} d u d s d v \\
& \leq k+c_{2} M+\min \left\{\left(1-c_{2}\right) M-k, k-c_{1} M-N\right\} \\
& \leq M, \quad x, y \in A(N, M), t \geq T, \\
\left(S_{1 k} x\right)(t)+\left(S_{2 k} y\right)(t) & \geq k-c_{1} M-\int_{T}^{+\infty} \int_{v}^{+\infty} \int_{s}^{+\infty} \frac{h(u)}{\alpha(s) \beta(v)} d u d s d v \\
& \geq k-c_{1} M-\min \left\{\left(1-c_{2}\right) M-k, k-c_{1} M-N\right\} \\
& \geq N, \quad x, y \in A(N, M), t \geq T,
\end{aligned}
$$

which infer that $S_{1 k}(A(N, M))+S_{2 k}(A(N, M)) \subseteq A(N, M)$ for any $x, y \in A(N, M)$.

Secondly, we show that $S_{1 k}$ is a contraction mapping. By (2.1), (2.2), and (2.6), we deduce that

$$
\left|\left(S_{1 k} x\right)(t)-\left(S_{1 k} y\right)(t)\right| \leq|p(t)||x(t-\tau)-y(t-\tau)| \leq\left(c_{1}+c_{2}\right)\|x-y\|
$$

for $x, y \in A(N, M)$ and $t \geq T$, which gives that

$$
\left\|S_{1 k} x-S_{1 k} y\right\| \leq\left(c_{1}+c_{2}\right)\|x-y\| .
$$


Thirdly, we show that $S_{2 k}$ is completely continuous. Let $x \in A(N, M)$ and $\left\{x_{m}\right\}_{m \geq 1} \subseteq$ $A(N, M)$ with $\lim _{m \rightarrow+\infty} x_{m}=x$. By $(2.7)$, we obtain that

$$
\begin{array}{r}
\left|\left(S_{2 k} x_{m}\right)(t)-\left(S_{2 k} x\right)(t)\right| \\
\leq \int_{T}^{+\infty} \int_{v}^{+\infty} \int_{s}^{+\infty} \frac{1}{\alpha(s) \beta(v)} \mid f\left(u, x_{m}\left(\sigma_{1}(u)\right), \ldots, x_{m}\left(\sigma_{n}(u)\right)\right) \\
-f\left(u, x\left(\sigma_{1}(u)\right), \ldots, x\left(\sigma_{n}(u)\right)\right) \mid d u d s d v, \\
t \geq T, m \geq 1 .
\end{array}
$$

Using (2.3) and (2.4), we conclude that

$$
\begin{aligned}
& \begin{array}{r}
\left|f\left(u, x_{m}\left(\sigma_{1}(u)\right), \ldots, x_{m}\left(\sigma_{n}(u)\right)\right)-f\left(u, x\left(\sigma_{1}(u)\right), \ldots, x\left(\sigma_{n}(u)\right)\right)\right| \leq 2 h(u), \\
u \in[T,+\infty), m \geq 1, \\
\int_{s}^{+\infty}\left|f\left(u, x_{m}\left(\sigma_{1}(u)\right), \ldots, x_{m}\left(\sigma_{n}(u)\right)\right)-f\left(u, x\left(\sigma_{1}(u)\right), \ldots, x\left(\sigma_{n}(u)\right)\right)\right| d u \leq 2 \int_{s}^{+\infty} h(u) d u, \\
\quad s \in[T, \infty), m \geq 1,
\end{array} \\
& \int_{v}^{+\infty} \int_{s}^{+\infty} \frac{1}{\alpha(s)}\left|f\left(u, x_{m}\left(\sigma_{1}(u)\right), \ldots, x_{m}\left(\sigma_{n}(u)\right)\right)-f\left(u, x\left(\sigma_{1}(u)\right), \ldots, x\left(\sigma_{n}(u)\right)\right)\right| d u d s \\
& \leq 2 \int_{v}^{+\infty} \int_{s}^{+\infty} \frac{h(u)}{\alpha(s)} d u d s, \quad v, s \in[T, \infty), m \geq 1 .
\end{aligned}
$$

In light of (2.11)-(2.12),

$$
\left|f\left(u, x_{m}\left(\sigma_{1}(u)\right), \ldots, x_{m}\left(\sigma_{n}(u)\right)\right)-f\left(u, x\left(\sigma_{1}(u)\right), \ldots, x\left(\sigma_{n}(u)\right)\right)\right| \longrightarrow 0 \quad \text { as } m \longrightarrow+\infty
$$

and the Lebesgue dominated convergence theorem, we conclude that

$$
\lim _{m \rightarrow+\infty}\left\|S_{2 k} x_{m}-S_{2 k} x\right\|=0
$$

which means that $S_{2 k}$ is continuous in $A(N, M)$.

Now we show that $S_{2 k}$ is completely continuous. By virtue of (2.3), (2.4), and (2.7), we get that

$$
\left\|S_{2 k} x\right\| \leq \int_{T}^{+\infty} \int_{v}^{+\infty} \int_{s}^{+\infty} \frac{h(u)}{\alpha(s) \beta(v)} d u d s d v, \quad x \in A(N, M)
$$


That is, $S_{2 k}(A(N, M))$ is uniform bounded. It follows from (2.4) that for each $\varepsilon>0$, there exists $T^{*}>T$ such that

$$
\int_{T^{*}}^{+\infty} \int_{v}^{+\infty} \int_{s}^{+\infty} \frac{h(u)}{\alpha(s) \beta(v)} d u d s d v<\frac{\varepsilon}{2}
$$

In view of (2.3), (2.7), and (2.16), we infer that

$$
\begin{aligned}
& \left|\left(S_{2 k} x\right)\left(t_{2}\right)-\left(S_{2 k} x\right)\left(t_{1}\right)\right| \\
& \quad \leq \int_{t_{2}}^{+\infty} \int_{v}^{+\infty} \int_{s}^{+\infty} \frac{h(u)}{\alpha(s) \beta(v)} d u d s d v+\int_{t_{1}}^{+\infty} \int_{v}^{+\infty} \int_{s}^{+\infty} \frac{h(u)}{\alpha(s) \beta(v)} d u d s d v \\
& \quad<\varepsilon, \quad x \in A(N, M), t_{2}>t_{1} \geq T^{*} .
\end{aligned}
$$

From (2.3) and (2.7), we get that

$$
\begin{aligned}
& \left|\left(S_{2 k} x\right)\left(t_{2}\right)-\left(S_{2 k} x\right)\left(t_{1}\right)\right| \\
& \quad \leq \int_{t_{1}}^{t_{2}} \int_{v}^{+\infty} \int_{s}^{+\infty} \frac{h(u)}{\alpha(s) \beta(v)} d u d s d v, \quad x \in A(N, M), T \leq t_{1} \leq t_{2} \leq T^{*},
\end{aligned}
$$

which together with (2.4) ensures that there exists $\delta>0$ satisfying

$$
\left|\left(S_{2 k} x\right)\left(t_{2}\right)-\left(S_{2 k} x\right)\left(t_{1}\right)\right|<\varepsilon, \quad x \in A(N, M), t_{1}, t_{2} \in\left[T, T^{*}\right] \text { with }\left|t_{2}-t_{1}\right|<\delta
$$

Clearly,

$$
\left|\left(S_{2 k} x\right)\left(t_{2}\right)-\left(S_{2 k} x\right)\left(t_{1}\right)\right|=0<\varepsilon, \quad x \in A(N, M), t_{0} \leq t_{1} \leq t_{2} \leq T .
$$

That is, $S_{2 k}(A(N, M))$ is equicontinuous on $\left[t_{0},+\infty\right)$. Consequently, $S_{2 k}$ is completely continuous. By Lemma 1.2, there is $x_{0} \in A(N, M)$ such that $S_{1} x_{0}+S_{2} x_{0}=x_{0}$, which is a bounded nonoscillatory solution of (1.7).

Lastly, we demonstrate that (1.7) possesses uncountably many bounded nonoscillatory solutions in $A(N, M)$. Let $k_{1}, k_{2} \in\left(c_{1} M+N,\left(1-c_{2}\right) M\right)$ and $k_{1} \neq k_{2}$. For each $j \in\{1,2\}$, we choose $T_{j}>T_{0}$ and the mappings $S_{j k_{1}}$ and $S_{j k_{2}}$ satisfying (2.5)-(2.7) with $k$ and $T$ replaced by $k_{j}$ and $T_{j}$, respectively, and some $T_{3}>\max \left\{T_{1}, T_{2}\right\}$ such that

$$
\int_{T_{3}}^{+\infty} \int_{v}^{+\infty} \int_{s}^{+\infty} \frac{h(u)}{\alpha(s) \beta(v)} d u d s d v<\frac{\left|k_{1}-k_{2}\right|}{2}
$$

Obviously, there are $x, y \in A(N, M)$ such that $S_{1 k_{1}} x+S_{2 k_{1}} x=x$ and $S_{1 k_{2}} y+S_{2 k_{2}} y=y$, respectively. That is, $x$ and $y$ are two bounded nonoscillatory solutions of $(1.7)$ in $A(N, M)$. 
In order to prove that (1.7) possesses uncountably many bounded nonoscillatory solutions in $A(N, M)$, we prove only that $x \neq y$. In fact, by (2.6) and (2.7), we gain that for $t \geq T_{3}$

$$
\begin{aligned}
& x(t)=k_{1}-p(t) x(t-\tau)+\int_{t}^{+\infty} \int_{v}^{+\infty} \int_{s}^{+\infty} \frac{1}{\alpha(s) \beta(v)} f\left(u, x\left(\sigma_{1}(u)\right), \ldots, x\left(\sigma_{n}(u)\right)\right) d u d s d v \\
& y(t)=k_{2}-p(t) y(t-\tau)+\int_{t}^{+\infty} \int_{v}^{+\infty} \int_{s}^{+\infty} \frac{1}{\alpha(s) \beta(v)} f\left(u, y\left(\sigma_{1}(u)\right), \ldots, y\left(\sigma_{n}(u)\right)\right) d u d s d v
\end{aligned}
$$

which together with (2.1)-(2.3) imply that

$$
\begin{aligned}
|x(t)-y(t)| \geq & \left|k_{1}-k_{2}\right|-|p(t)||x(t-\tau)-y(t-\tau)|-\int_{t}^{+\infty} \int_{v}^{+\infty} \int_{s}^{+\infty} \frac{1}{\alpha(s) \beta(v)} \\
& \times\left|f\left(u, x\left(\sigma_{1}(u)\right), \ldots, x\left(\sigma_{n}(u)\right)\right)-f\left(u, y\left(\sigma_{1}(u)\right), \ldots, y\left(\sigma_{n}(u)\right)\right)\right| d u d s d v \\
\geq & \left|k_{1}-k_{2}\right|-\left(c_{1}+c_{2}\right)\|x-y\| \\
& -2 \int_{T_{3}}^{+\infty} \int_{v}^{+\infty} \int_{s}^{+\infty} \frac{h(u)}{\alpha(s) \beta(v)} d u d s d v, \quad t \geq T_{3}
\end{aligned}
$$

which means that

$$
\|x-y\| \geq \frac{1}{1+c_{1}+c_{2}}\left(\left|k_{1}-k_{2}\right|-2 \int_{T_{3}}^{+\infty} \int_{v}^{+\infty} \int_{s}^{+\infty} \frac{h(u)}{\alpha(s) \beta(v)} d u d s d v\right)>0
$$

by (2.1) and (2.21). That is, $x \neq y$. This completes the proof.

Theorem 2.2. Assume that there exist constants $M, N, c_{1}, c_{2}, T_{0}$ and a function $h \in C\left(\left[t_{0},+\infty\right), \mathbb{R}^{+}\right)$ satisfying (2.3), (2.4) and

$$
\begin{gathered}
0<\left(1-c_{2}\right) N<\left(1-c_{1}\right) M ; \\
0 \leq c_{2} \leq p(t) \leq c_{1}<1, \quad t \geq T_{0}>t_{0} .
\end{gathered}
$$

Then (1.7) possesses uncountably many bounded nonoscillatory solutions in $A(N, M)$.

Proof. Set $k \in\left(c_{1} M+N, M+c_{2} N\right)$. It follows from (2.4) that there exists $T>T_{0}$ such that

$$
\int_{T}^{+\infty} \int_{v}^{+\infty} \int_{s}^{+\infty} \frac{h(u)}{\alpha(s) \beta(v)} d u d s d v<\min \left\{M+c_{2} N-k, k-c_{1} M-N\right\}
$$


Define two mappings $S_{1 k}$ and $S_{2 k}: A(N, M) \rightarrow C\left(\left[t_{0},+\infty\right), \mathbb{R}\right)$ by (2.6) and (2.7), respectively. By virtue of (2.3), (2.6), (2.7), (2.26), and (2.27), we obtain that

$$
\begin{aligned}
\left(S_{1 k} x\right)(t)+\left(S_{2 k} y\right)(t) & \leq k-p(t) x(t-\tau)+\int_{T}^{+\infty} \int_{v}^{+\infty} \int_{s}^{+\infty} \frac{h(u)}{\alpha(s) \beta(v)} d u d s d v \\
& \leq k-c_{2} N+\min \left\{M+c_{2} N-k, k-c_{1} M-N\right\} \\
& \leq M, \quad x, y \in A(N, M), t \geq T, \\
\left(S_{1 k} x\right)(t)+\left(S_{2 k} y\right)(t) & \geq k-p(t) x(t-\tau)-\int_{T}^{+\infty} \int_{v}^{+\infty} \int_{s}^{+\infty} \frac{h(u)}{\alpha(s) \beta(v)} d u d s d v \\
& \geq k-c_{1} M-\min \left\{M+c_{2} N-k, k-c_{1} M-N\right\} \\
& \geq N, \quad x, y \in A(N, M), t \geq T,
\end{aligned}
$$

which yield that $S_{1 k}(A(N, M))+S_{2 k}(A(N, M)) \subseteq A(N, M)$.

By a similar argument used in the proof of Theorem 2.1, we gain that $S_{1 k}$ is a contraction mapping $S_{2 k}$ is completely continuous, and (1.7) possesses uncountably many nonoscillatory solutions. This completes the proof.

Theorem 2.3. Assume that there exist constants $M, N, c_{1}, c_{2}, T_{0}$ and a function $h \in C\left(\left[t_{0},+\infty\right), \mathbb{R}^{+}\right)$ satisfying (2.3), (2.4), (2.25), and

$$
-1<-c_{1} \leq p(t) \leq-c_{2} \leq 0, \quad t \geq T_{0}>t_{0} .
$$

Then (1.7) possesses uncountably many bounded nonoscillatory solutions in $A(N, M)$.

Proof. Set $k \in\left(\left(1-c_{2}\right) N,\left(1-c_{1}\right) M\right)$. It follows from (2.4) that there exists $T>T_{0}$ satisfying

$$
\int_{T}^{+\infty} \int_{v}^{+\infty} \int_{s}^{+\infty} \frac{h(u)}{\alpha(s) \beta(v)} d u d s d v<\min \left\{\left(1-c_{1}\right) M-k, k-\left(1-c_{2}\right) N\right\} .
$$

Let $S_{1 k}$ and $S_{2 k}: A(N, M) \rightarrow C\left(\left[t_{0},+\infty\right), \mathbb{R}\right)$ be defined by (2.6) and (2.7), respectively. In view of (2.3), (2.6), (2.7), (2.30), and (2.31), we obtain that

$$
\begin{aligned}
\left(S_{1} x\right)(t)+\left(S_{2} y\right)(t) & \leq k-p(t) x(t-\tau)+\int_{T}^{+\infty} \int_{v}^{+\infty} \int_{s}^{+\infty} \frac{h(u)}{\alpha(s) \beta(v)} d u d s d v \\
& \leq k+c_{1} M+\min \left\{\left(1-c_{1}\right) M-k, k-\left(1-c_{2}\right) N\right\} \\
& \leq M, \quad x, y \in A(N, M), t \geq T, \\
\left(S_{1} x\right)(t)+\left(S_{2} y\right)(t) & \geq k-p(t) x(t-\tau)-\int_{T}^{+\infty} \int_{v}^{+\infty} \int_{s}^{+\infty} \frac{h(u)}{\alpha(s) \beta(v)} d u d s d v \\
& \geq k+c_{2} N-\min \left\{\left(1-c_{1}\right) M-k, k-\left(1-c_{2}\right) N\right\} \\
& \geq N, \quad x, y \in A(N, M), t \geq T,
\end{aligned}
$$

which mean that $S_{1 k}(A(N, M))+S_{2 k}(A(N, M)) \subseteq A(N, M)$. 
The rest of the proof is similar to the proof of Theorem 2.1 and is omitted. This completes the proof.

Theorem 2.4. Assume that there exist constants $M$ and $N$ with $M>N>0$ and a function $h \in$ $C\left(\left[t_{0},+\infty\right), \mathbb{R}^{+}\right)$satisfying (2.3) and (2.4). If $p(t)=1$ for each $t \in\left[t_{0},+\infty\right)$, then (1.7) possesses uncountably many bounded nonoscillatory solutions in $A(N, M)$.

Proof. Set $k \in(N, M)$. It follows from (2.4) that there exists $T \geq t_{0}+\tau$ satisfying

$$
\int_{T}^{+\infty} \int_{v}^{+\infty} \int_{s}^{+\infty} \frac{h(u)}{\alpha(s) \beta(v)} d u d s d v<\min \{M-k, k-N\}
$$

Define a mapping $S_{k}: A(N, M) \rightarrow C\left(\left[t_{0},+\infty\right), \mathbb{R}\right)$ by

$\left(S_{k} x\right)(t)= \begin{cases}k+\sum_{i=1}^{\infty} \int_{t+(2 i-1) \tau}^{t+2 i \tau} \int_{v}^{+\infty} \int_{s}^{+\infty} \frac{1}{\alpha(s) \beta(v)} f\left(u, x\left(\sigma_{1}(u)\right), \ldots, x\left(\sigma_{n}(u)\right)\right) d u d s d v, & t \geq T, \\ \left(S_{k} x\right)(T), & t_{0} \leq t<T\end{cases}$

for $x \in A(N, M)$.

Firstly, we prove that $S_{k}(A(N, M)) \subseteq A(N, M)$. By virtue of (2.3), (2.33) and (2.34), we obtain that

$$
\begin{aligned}
\left(S_{k} x\right)(t) & \leq k+\sum_{i=1}^{\infty} \int_{t+(2 i-1) \tau}^{t+2 i \tau} \int_{v}^{+\infty} \int_{s}^{+\infty} \frac{h(u)}{\alpha(s) \beta(v)} d u d s d v \\
& \leq k+\int_{T}^{+\infty} \int_{v}^{+\infty} \int_{s}^{+\infty} \frac{h(u)}{\alpha(s) \beta(v)} d u d s d v \\
& \leq M, \quad x \in A(N, M), t \geq T \\
\left(S_{k} x\right)(t) & \geq k-\sum_{i=1}^{\infty} \int_{t+(2 i-1) \tau}^{t+2 i \tau} \int_{v}^{+\infty} \int_{s}^{+\infty} \frac{h(u)}{\alpha(s) \beta(v)} d u d s d v \\
& \geq k-\int_{T}^{+\infty} \int_{v}^{+\infty} \int_{s}^{+\infty} \frac{h(u)}{\alpha(s) \beta(v)} d u d s d v \\
& \geq N, \quad x \in A(N, M), t \geq T
\end{aligned}
$$

which imply that $S_{k}(A(N, M)) \subseteq A(N, M)$. 
Secondly, we show that $S_{k}$ is continuous in $A(N, M)$. Let $x \in A(N, M)$ and $\left\{x_{m}\right\}_{m \geq 1} \subseteq$ $A(N, M)$ with $\lim _{m \rightarrow+\infty} x_{m}=x$. By (2.34), we get that

$$
\begin{aligned}
& \left|\left(S_{k} x_{m}\right)(t)-\left(S_{k} x\right)(t)\right| \\
& \leq \sum_{i=1}^{\infty} \int_{t+(2 i-1) \tau}^{t+2 i \tau} \int_{v}^{+\infty} \int_{s}^{+\infty} \frac{1}{\alpha(s) \beta(v)} \mid f\left(u, x_{m}\left(\sigma_{1}(u)\right), \ldots, x_{m}\left(\sigma_{n}(u)\right)\right) \\
& -f\left(u, x\left(\sigma_{1}(u)\right), \ldots, x\left(\sigma_{n}(u)\right)\right) \mid d u d s d v \\
& \leq \int_{T}^{+\infty} \int_{v}^{+\infty} \int_{s}^{+\infty} \frac{1}{\alpha(s) \beta(v)} \mid f\left(u, x_{m}\left(\sigma_{1}(u)\right), \ldots, x_{m}\left(\sigma_{n}(u)\right)\right) \\
& -f\left(u, x\left(\sigma_{1}(u)\right), \ldots, x\left(\sigma_{n}(u)\right)\right) \mid d u d s d v, \quad t \geq T, m \geq 1 .
\end{aligned}
$$

In view of (2.12), (2.13), (2.36), and the Lebesgue dominated convergence theorem, we deduce that

$$
\lim _{m \rightarrow+\infty}\left\|S_{k} x_{m}-S_{k} x\right\|=0,
$$

which means that $S_{k}$ is continuous in $A(N, M)$.

Thirdly, we show that $S_{k}(A(N, M))$ is relatively compact. From (2.3), (2.33), and (2.34), we gain that

$$
\left\|S_{k} x\right\| \leq k+\int_{T}^{+\infty} \int_{v}^{+\infty} \int_{s}^{+\infty} \frac{h(u)}{\alpha(s) \beta(v)} d u d s d v \leq 2 M, \quad x \in A(N, M),
$$

which means that $S_{k}(A(N, M))$ is uniform bounded.

Let $\varepsilon>0$. It follows from (2.4) that there exist $\bar{T}>T^{*}>T$ such that

$$
\begin{aligned}
& \int_{T^{*}}^{+\infty} \int_{v}^{+\infty} \int_{s}^{+\infty} \frac{h(u)}{\alpha(s) \beta(v)} d u d s d v<\frac{\varepsilon}{2} \\
& \int_{\bar{T}}^{+\infty} \int_{v}^{+\infty} \int_{s}^{+\infty} \frac{h(u)}{\alpha(s) \beta(v)} d u d s d v<\frac{\varepsilon}{4}
\end{aligned}
$$

By (2.3), (2.34), and (2.39), we deduce that

$$
\begin{aligned}
\left|\left(S_{k} x\right)\left(t_{2}\right)-\left(S_{k} x\right)\left(t_{1}\right)\right| & \leq \sum_{i=1}^{\infty}\left(\int_{t_{2}+(2 i-1) \tau}^{t_{2}+2 i \tau}+\int_{t_{1}+(2 i-1) \tau}^{t_{1}+2 i \tau}\right) \int_{v}^{+\infty} \int_{s}^{+\infty} \frac{h(u)}{\alpha(s) \beta(v)} d u d s d v \\
& \leq 2 \int_{T^{*}}^{+\infty} \int_{v}^{+\infty} \int_{s}^{+\infty} \frac{h(u)}{\alpha(s) \beta(v)} d u d s d v \\
& <\varepsilon, \quad x \in A(N, M), t_{2}>t_{1} \geq T^{*}
\end{aligned}
$$


Choose an integer $z \geq 1$ with $T+(2 z+1) \tau \geq \bar{T}$, and put

$$
\begin{gathered}
A=\max \left\{\int_{v}^{+\infty} \int_{s}^{+\infty} \frac{h(u)}{\alpha(s) \beta(v)} d u d s: v \in\left[T+\tau, T^{*}+(2 z-1) \tau\right]\right\}, \\
B=\max \left\{\int_{v}^{+\infty} \int_{s}^{+\infty} \frac{h(u)}{\alpha(s) \beta(v)} d u d s: v \in\left[T+2 \tau, T^{*}+2 z \tau\right]\right\}, \\
\delta=\min \left\{\frac{\varepsilon}{1+4 A z}, \frac{\varepsilon}{1+4 B z}\right\} .
\end{gathered}
$$

Equation (2.4) means that $\max \{A, B\}<+\infty$. It follows from (2.3), (2.34), and (2.40)-(2.42) that for $x \in A(N, M), t_{1}, t_{2} \in\left[T, T^{*}\right]$ with $t_{1} \leq t_{2} \leq t_{1}+\delta$

$$
\begin{aligned}
\left|\left(S_{k} x\right)\left(t_{2}\right)-\left(S_{k} x\right)\left(t_{1}\right)\right| & \mid \sum_{i=1}^{z}\left(\int_{t_{2}+(2 i-1) \tau}^{t_{2}+2 i \tau}-\int_{t_{1}+(2 i-1) \tau}^{t_{1}+2 i \tau}\right) \\
& \times \int_{v}^{+\infty} \int_{s}^{+\infty} \frac{1}{\alpha(s) \beta(v)} f\left(u, x\left(\sigma_{1}(u)\right), \ldots, x\left(\sigma_{n}(u)\right)\right) d u d s d v \mid \\
& +\sum_{i=z+1}^{\infty}\left(\int_{t_{2}+(2 i-1) \tau}^{t_{2}+2 i \tau}+\int_{t_{1}+(2 i-1) \tau}^{t_{1}+2 i \tau}\right) \int_{v}^{+\infty} \int_{s}^{+\infty} \frac{h(u)}{\alpha(s) \beta(v)} d u d s d v \\
\leq & \mid \sum_{i=1}^{z}\left(\int_{t_{2}+(2 i-1) \tau}^{t_{1}+(2 i-1) \tau}+\int_{t_{1}+(2 i-1) \tau}^{t_{2}+2 i \tau}+\int_{t_{1}+2 i \tau}^{t_{1}+(2 i-1) \tau}\right) \\
& \times \int_{v}^{+\infty} \int_{s}^{+\infty} \frac{1}{\alpha(s) \beta(v)} f\left(u, x\left(\sigma_{1}(u)\right), \ldots, x\left(\sigma_{n}(u)\right)\right) d u d s d v \mid \\
& +2 \int_{\bar{T}}^{+\infty} \int_{v}^{+\infty} \int_{s}^{+\infty} \frac{h(u)}{\alpha(s) \beta(v)} d u d s d v \\
\leq & \sum_{i=1}^{z}\left(\int_{t_{1}+(2 i-1) \tau}^{t_{2}+(2 i-1) \tau}+\int_{t_{1}+2 i \tau}^{t_{2}+2 i \tau}\right) \int_{v}^{+\infty} \int_{s}^{+\infty} \frac{h(u)}{\alpha(s) \beta(v)} d u d s d v+\frac{\varepsilon}{2} \\
\leq & A z\left(t_{2}-t_{1}\right)+B z\left(t_{2}-t_{1}\right)+\frac{\varepsilon}{2} \\
< & \varepsilon .
\end{aligned}
$$

It is not difficult to verify that

$$
\left|\left(S_{k} x\right)\left(t_{2}\right)-\left(S_{k} x\right)\left(t_{1}\right)\right|=0<\varepsilon, \quad x \in A(N, M), t_{0} \leq t_{1} \leq t_{2} \leq T .
$$


Therefore $S_{k}(A(N, M))$ is equicontinuous on $\left[t_{0},+\infty\right)$, and consequently $S_{k}$ is relatively compact. By Lemma 1.3, there is $x_{0} \in A(N, M)$ such that $S x_{0}=x_{0}$, which together with (2.34) yields that for $t \geq T+\tau$

$$
\begin{aligned}
& x_{0}(t)=k+\sum_{i=1}^{\infty} \int_{t+(2 i-1) \tau}^{t+2 i \tau} \int_{v}^{+\infty} \int_{s}^{+\infty} \frac{1}{\alpha(s) \beta(v)} f\left(u, x_{0}\left(\sigma_{1}(u)\right), \ldots, x_{0}\left(\sigma_{n}(u)\right)\right) d u d s d v \\
& x_{0}(t-\tau)=k+\sum_{i=1}^{\infty} \int_{t+2(i-1) \tau}^{t+(2 i-1) \tau} \int_{v}^{+\infty} \int_{s}^{+\infty} \frac{1}{\alpha(s) \beta(v)} f\left(u, x_{0}\left(\sigma_{1}(u)\right), \ldots, x_{0}\left(\sigma_{n}(u)\right)\right) d u d s d v
\end{aligned}
$$

which mean that

$$
x_{0}(t)+x_{0}(t-\tau)=2 k+\int_{t}^{+\infty} \int_{v}^{+\infty} \int_{s}^{+\infty} \frac{1}{\alpha(s) \beta(v)} f\left(u, x_{0}\left(\sigma_{1}(u)\right), \ldots, x_{0}\left(\sigma_{n}(u)\right)\right) d u d s d v
$$

It is easy to show that $x_{0}$ is a bounded nonoscillatory solution of (1.7).

Finally, we demonstrate that (1.7) possesses uncountably many bounded nonoscillatory solutions in $A(N, M)$. Let $k_{1}, k_{2} \in(N, M)$ and $k_{1} \neq k_{2}$. For each $j \in\{1,2\}$, we pick up a positive integer $T_{j} \geq t_{0}+\tau$ and the mapping $S_{k_{j}}$ satisfying (2.33) and (2.34), where $k$ and $T$ are replaced by $k_{j}$ and $T_{j}$, respectively, and some $T_{3}>\max \left\{T_{1}, T_{2}\right\}$ satisfying (2.21). Clearly, there are $x$ and $y \in A(N, M)$ such that $S_{k_{1}} x=x$ and $S_{k_{2}} y=y$, respectively. That is, $x$ and $y$ are bounded nonoscillatory solutions of (1.7) in $A(N, M)$. By (2.34) we get that for $t \geq T_{3}$

$$
\begin{aligned}
& x(t)=k_{1}+\sum_{i=1}^{\infty} \int_{t+(2 i-1) \tau}^{t+2 i \tau} \int_{v}^{+\infty} \int_{s}^{+\infty} \frac{1}{\alpha(s) \beta(v)} f\left(u, x\left(\sigma_{1}(u)\right), \ldots, x\left(\sigma_{n}(u)\right)\right) d u d s d v \\
& y(t)=k_{2}+\sum_{i=1}^{\infty} \int_{t+(2 i-1) \tau}^{t+2 i \tau} \int_{v}^{+\infty} \int_{s}^{+\infty} \frac{1}{\alpha(s) \beta(v)} f\left(u, y\left(\sigma_{1}(u)\right), \ldots, y\left(\sigma_{n}(u)\right)\right) d u d s d v,
\end{aligned}
$$

which together with (2.3) and (2.21) yield that

$$
\|x-y\| \geq\left|k_{1}-k_{2}\right|-2 \int_{T_{3}}^{+\infty} \int_{v}^{+\infty} \int_{s}^{+\infty} \frac{h(u)}{\alpha(s) \beta(v)} d u d s d v>0
$$

which implies that $x \neq y$. This completes the proof.

Theorem 2.5. Assume that there exist constants $M$ and $N$ with $M>N>0$ and a function $h \in$ $C\left(\left[t_{0},+\infty\right), \mathbb{R}^{+}\right)$satisfying $(2.3)$ and

$$
\sum_{i=1}^{\infty} \int_{t_{0}+i \tau}^{+\infty} \int_{v}^{+\infty} \int_{s}^{+\infty} \frac{h(u)}{\alpha(s) \beta(v)} d u d s d v<+\infty
$$

If $p(t)=-1$ for each $t \in\left[t_{0},+\infty\right)$, then (1.7) possesses uncountably many bounded nonoscillatory solutions in $A(N, M)$. 
Proof. Set $k \in(N, M)$. It follows from (2.49) that there exists $T>t_{0}$ satisfying

$$
\sum_{i=1}^{\infty} \int_{T+i \tau}^{+\infty} \int_{v}^{+\infty} \int_{s}^{+\infty} \frac{h(u)}{\alpha(s) \beta(v)} d u d s d v<\min \{M-k, k-N\}
$$

Define a mapping $S_{k}: A(N, M) \rightarrow C\left(\left[t_{0},+\infty\right), \mathbb{R}\right)$ by

$$
\left(S_{k} x\right)(t)= \begin{cases}k-\sum_{i=1}^{\infty} \int_{t+i \tau}^{+\infty} \int_{v}^{+\infty} \int_{s}^{+\infty} \frac{1}{\alpha(s) \beta(v)} f\left(u, x\left(\sigma_{1}(u)\right), \ldots, x\left(\sigma_{n}(u)\right)\right) d u d s d v, & t \geq T, \\ \left(S_{k} x\right)(T), & t_{0} \leq t<T\end{cases}
$$

for $x \in A(N, M)$.

Firstly, we prove that $S_{k}(A(N, M)) \subseteq A(N, M)$. By (2.3), (2.50), and (2.51), we obtain that

$$
\begin{array}{ll}
\left(S_{k} x\right)(t) \leq k+\sum_{i=1}^{\infty} \int_{t+i \tau}^{+\infty} \int_{v}^{+\infty} \int_{s}^{+\infty} \frac{h(u)}{\alpha(s) \beta(v)} d u d s d v \leq M, & x \in A(N, M), t \geq T, \\
\left(S_{k} x\right)(t) \geq k-\sum_{i=1}^{\infty} \int_{t+i \tau}^{+\infty} \int_{v}^{+\infty} \int_{s}^{+\infty} \frac{h(u)}{\alpha(s) \beta(v)} d u d s d v \geq N, & x \in A(N, M), t \geq T,
\end{array}
$$

which imply that $S(A(N, M)) \subseteq A(N, M)$.

Secondly, we show that $S_{k}$ is continuous in $A(N, M)$. Let $x \in A(N, M)$ and $\left\{x_{m}\right\}_{m \geq 1} \subseteq$ $A(N, M)$ with $\lim _{m \rightarrow+\infty} x_{m}=x$. By $(2.51)$, we get that

$$
\begin{aligned}
& \left|\left(S_{k} x_{m}\right)(t)-\left(S_{k} x\right)(t)\right| \\
& \leq \sum_{i=1}^{\infty} \int_{T+i \tau}^{+\infty} \int_{v}^{+\infty} \int_{s}^{+\infty} \frac{1}{\alpha(s) \beta(v)} \mid f\left(u, x_{m}\left(\sigma_{1}(u)\right), \ldots, x_{m}\left(\sigma_{n}(u)\right)\right) \\
& \quad-f\left(u, x\left(\sigma_{1}(u)\right), \ldots, x\left(\sigma_{n}(u)\right)\right) \mid d u d s d v, \quad t \geq T, m \geq 1 .
\end{aligned}
$$

Equation (2.53) together with (2.3), (2.49) and the Lebesgue dominated convergence theorem yields that

$$
\lim _{m \rightarrow+\infty}\left\|S_{k} x_{m}-S_{k} x\right\|=0,
$$

that is, $S_{k}$ is continuous in $A(N, M)$.

Thirdly, we show that $S_{k}(A(N, M))$ is relatively compact. From (2.3), (2.50), and (2.51), we obtain that

$$
\left\|S_{k} x\right\| \leq k+\sum_{i=1}^{\infty} \int_{T+i \tau}^{+\infty} \int_{v}^{+\infty} \int_{s}^{+\infty} \frac{h(u)}{\alpha(s) \beta(v)} d u d s d v \leq 2 M, \quad x \in A(N, M),
$$


which means that $S_{k}(A(N, M))$ is uniform bounded. It follows from (2.49) that, for each $\varepsilon>0$, there exists $T^{*}>T$ such that

$$
\sum_{i=1}^{\infty} \int_{T^{*}+i \tau}^{+\infty} \int_{v}^{+\infty} \int_{s}^{+\infty} \frac{h(u)}{\alpha(s) \beta(v)} d u d s d v<\frac{\varepsilon}{2}
$$

Notice that (2.3), (2.51), and (2.56) yield that

$$
\begin{aligned}
\left|\left(S_{k} x\right)\left(t_{2}\right)-\left(S_{k} x\right)\left(t_{1}\right)\right| & \leq 2 \sum_{i=1}^{\infty} \int_{T^{*}+i \tau}^{+\infty} \int_{v}^{+\infty} \int_{s}^{+\infty} \frac{h(u)}{\alpha(s) \beta(v)} d u d s d v \\
& <\varepsilon, \quad x \in A(N, M), t_{2}>t_{1} \geq T^{*}
\end{aligned}
$$

Choose a positive integer $z$ satisfying $T+z \tau \geq T^{*}$. Put

$$
\begin{gathered}
D=\max \left\{\int_{v}^{+\infty} \int_{s}^{+\infty} \frac{h(u)}{\alpha(s) \beta(v)} d u d s: v \in\left[T+\tau, T^{*}+z \tau\right]\right\}, \\
\delta=\frac{\varepsilon}{1+2 D z} .
\end{gathered}
$$

By (2.3), (2.51), and (2.58), we gain that

$$
\begin{aligned}
& \left|\left(S_{k} x\right)\left(t_{2}\right)-\left(S_{k} x\right)\left(t_{1}\right)\right| \\
& \quad=\left|\sum_{i=1}^{\infty}\left(\int_{t_{2}+i \tau}^{+\infty}-\int_{t_{1}+i \tau}^{+\infty}\right) \int_{v}^{+\infty} \int_{s}^{+\infty} \frac{1}{\alpha(s) \beta(v)} f\left(u, x\left(\sigma_{1}(u)\right), \ldots, x\left(\sigma_{n}(u)\right)\right) d u d s d v\right| \\
& \quad \leq \sum_{i=1}^{\infty} \int_{t_{1}+i \tau}^{t_{2}+i \tau} \int_{v}^{+\infty} \int_{s}^{+\infty} \frac{h(u)}{\alpha(s) \beta(v)} d u d s d v \\
& \quad \leq \sum_{i=1}^{z} \int_{t_{1}+i \tau}^{t_{2}+i \tau} \int_{v}^{+\infty} \int_{s}^{+\infty} \frac{h(u)}{\alpha(s) \beta(v)} d u d s d v+\sum_{i=z+1}^{\infty} \int_{t_{1}+i \tau}^{t_{2}+i \tau} \int_{v}^{+\infty} \int_{s}^{+\infty} \frac{h(u)}{\alpha(s) \beta(v)} d u d s d v \\
& \quad \leq D z\left(t_{2}-t_{1}\right)+\sum_{i=1}^{\infty} \int_{T^{*}+i \tau}^{+\infty} \int_{v}^{+\infty} \int_{s}^{+\infty} \frac{h(u)}{\alpha(s) \beta(v)} d u d s d v \\
& \quad<\varepsilon, \quad x \in A(N, M), t_{1}, t_{2} \in\left[T, T^{*}\right] \text { with } t_{1} \leq t_{2} \leq t_{1}+\delta .
\end{aligned}
$$

Clearly,

$$
\left|\left(S_{k} x\right)\left(t_{2}\right)-\left(S_{k} x\right)\left(t_{1}\right)\right|=0<\varepsilon, \quad x \in A(N, M), t_{0} \leq t_{1} \leq t_{2} \leq T .
$$

That is, $S_{k}(A(N, M))$ is equicontinuous on $\left[t_{0},+\infty\right)$, and $S_{k}$ is relatively compact. The rest argument is similar to the proof of Theorem 2.4 and is omitted. This completes the proof. 
Theorem 2.6. Assume that there exist constants $M, N, c_{1}, c_{2}, T_{0}$ and a function $h \in C\left(\left[t_{0},+\infty\right), \mathbb{R}^{+}\right)$ satisfying (2.3), (2.4), and

$$
\begin{gathered}
1<c_{2}<c_{1}<c_{2}^{2}, \quad 0<\frac{c_{1}^{2}-c_{2}}{c_{1}} N<\frac{c_{2}^{2}-c_{1}}{c_{2}} M ; \\
c_{2} \leq p(t) \leq c_{1}, \quad t \geq T_{0} \geq t_{0}+\tau .
\end{gathered}
$$

Then (1.7) possesses uncountably many bounded nonoscillatory solutions in $A(N, M)$.

Proof. Set $k \in\left(c_{1} N+\left(c_{1} / c_{2}\right) M, c_{2} M+\left(c_{2} / c_{1}\right) N\right)$. It follows from (2.4) that there exists $T>T_{0}$ such that

$$
\int_{T}^{+\infty} \int_{v}^{+\infty} \int_{s}^{+\infty} \frac{h(u)}{\alpha(s) \beta(v)} d u d s d v<\min \left\{c_{2} M+\frac{c_{2}}{c_{1}} N-k, \frac{c_{2}}{c_{1}} k-M-c_{2} N\right\}
$$

Define two mappings $S_{1 k}$ and $S_{2 k}: A(N, M) \rightarrow C\left(\left[t_{0},+\infty\right), \mathbb{R}\right)$ by

$$
\begin{gathered}
\left(S_{1 k} x\right)(t)= \begin{cases}\frac{k}{p(t+\tau)}-\frac{x(t+\tau)}{p(t+\tau)}, & t \geq T, \\
\left(S_{1 k} x\right)(T), & t_{0} \leq t<T,\end{cases} \\
\left(S_{2 k} x\right)(t)= \begin{cases}\frac{1}{p(t+\tau)} \int_{t+\tau}^{+\infty} \int_{v}^{+\infty} \int_{s}^{+\infty} \frac{1}{\alpha(s) \beta(v)} f\left(u, x\left(\sigma_{1}(u)\right), \ldots, x\left(\sigma_{n}(u)\right)\right) d u d s d v, & t \geq T, \\
\left(S_{2 k} x\right)(T), & t_{0} \leq t<T\end{cases}
\end{gathered}
$$

for $x \in A(N, M)$.

Firstly, we show that $S_{1 k}(A(N, M))+S_{2 k}(A(N, M)) \subseteq A(N, M)$. From (2.3), (2.61)(2.65), we get that

$$
\begin{aligned}
& \left(S_{1 k} x\right)(t)+\left(S_{2 k} y\right)(t) \\
& \leq \frac{k}{c_{2}}-\frac{N}{c_{1}}+\frac{1}{c_{2}} \min \left\{c_{2} M+\frac{c_{2}}{c_{1}} N-k, \frac{c_{2}}{c_{1}} k-M-c_{2} N\right\} \\
& \leq M, \quad x, y \in A(N, M), t \geq T, \\
& \left(S_{1 k} x\right)(t)+\left(S_{2 k} y\right)(t) \\
& \quad \geq \frac{k}{c_{1}}-\frac{M}{c_{2}}-\frac{1}{c_{2}} \min \left\{c_{2} M+\frac{c_{2}}{c_{1}} N-k, \frac{c_{2}}{c_{1}} k-M-c_{2} N\right\} \\
& \geq N, \quad x, y \in A(N, M), t \geq T,
\end{aligned}
$$

That is, $S_{1 k}(A(N, M))+S_{2 k}(A(N, M)) \subseteq A(N, M)$. 
Secondly, by (2.61), (2.62), and (2.64), we conclude that

$$
\left\|S_{1 k} x-S_{1 k} y\right\| \leq \frac{1}{c_{2}}\|x-y\|, \quad x, y \in A(N, M),
$$

which implies that $S_{1 k}$ is a contraction mapping in $A(N, M)$.

Thirdly, we show that $S_{2 k}$ is completely continuous. Let $x \in A(N, M)$ and $\left\{x_{m}\right\}_{m \geq 1} \subseteq$ $A(N, M)$ be such that $x_{m} \rightarrow x$ as $m \rightarrow+\infty$. By (2.61), (2.62), and (2.65), we gain that

$$
\begin{aligned}
& \left|\left(S_{2 k} x_{m}\right)(t)-\left(S_{2 k} x\right)(t)\right| \\
& \begin{array}{r}
\leq \frac{1}{p(t+\tau)} \int_{t+\tau}^{+\infty} \int_{v}^{+\infty} \int_{s}^{+\infty} \frac{1}{\alpha(s) \beta(v)} \mid f\left(u, x_{m}\left(\sigma_{1}(u)\right), \ldots, x_{m}\left(\sigma_{n}(u)\right)\right) \\
-f\left(u, x\left(\sigma_{1}(t)\right), \ldots, x\left(\sigma_{n}(t)\right)\right) \mid d u d s d v \\
\leq \frac{1}{c_{2}} \int_{T}^{+\infty} \int_{v}^{+\infty} \int_{s}^{+\infty} \frac{1}{\alpha(s) \beta(v)} \mid f\left(u, x_{m}\left(\sigma_{1}(u)\right), \ldots, x_{m}\left(\sigma_{n}(u)\right)\right) \\
-f\left(u, x\left(\sigma_{1}(u)\right), \ldots, x\left(\sigma_{n}(u)\right)\right) \mid d u d s d v, \quad t \geq T, m \geq 1 .
\end{array}
\end{aligned}
$$

In view of (2.12), (2.13), (2.68), and the Lebesgue dominated convergence theorem, we obtain that

$$
\lim _{m \rightarrow+\infty}\left\|S_{2 k} x_{m}-S_{2 k} x\right\|=0
$$

that is, $S_{2 k}$ is continuous in $A(N, M)$.

For each $\varepsilon>0$, it follows from (2.4) that there exists $T^{*} \geq T$ satisfying

$$
\int_{T^{*}}^{+\infty} \int_{v}^{+\infty} \int_{s}^{+\infty} \frac{h(u)}{\alpha(s) \beta(v)} d u d s d v<\min \left\{\frac{c_{2} \varepsilon}{2}, \frac{c_{2}^{2} \varepsilon}{4 c_{1}}\right\}
$$

From (2.3), (2.61), (2.62), (2.65), and (2.70), we gain that

$$
\begin{aligned}
& \left|\left(S_{2 k} x\right)\left(t_{2}\right)-\left(S_{2 k} x\right)\left(t_{1}\right)\right| \\
& \quad \leq \frac{1}{c_{2}} \int_{t_{2}+\tau}^{+\infty} \int_{v}^{+\infty} \int_{s}^{+\infty} \frac{h(u)}{\alpha(s) \beta(v)} d u d s d v+\frac{1}{c_{2}} \int_{t_{1}+\tau}^{+\infty} \int_{v}^{+\infty} \int_{s}^{+\infty} \frac{h(u)}{\alpha(s) \beta(v)} d u d s d v \\
& \quad \leq \frac{2}{c_{2}} \int_{T^{*}}^{+\infty} \int_{v}^{+\infty} \int_{s}^{+\infty} \frac{h(u)}{\alpha(s) \beta(v)} d u d s d v \\
& \quad<\varepsilon, \quad x \in A(N, M), t_{2}>t_{1} \geq T^{*} .
\end{aligned}
$$


By (2.3), (2.61), (2.62), (2.65), and (2.70), we obtain that, for $x \in A(N, M), T \leq t_{1} \leq t_{2} \leq T^{*}$,

$$
\begin{aligned}
& \left|\left(S_{2 k} x\right)\left(t_{2}\right)-\left(S_{2 k} x\right)\left(t_{1}\right)\right| \\
& \leq \frac{1}{p\left(t_{2}+\tau\right)} \int_{t_{1}+\tau}^{t_{2}+\tau} \int_{v}^{+\infty} \int_{s}^{+\infty} \frac{h(u)}{\alpha(s) \beta(v)} d u d s d v \\
& \quad+\left|\frac{1}{p\left(t_{2}+\tau\right)}-\frac{1}{p\left(t_{1}+\tau\right)}\right| \int_{t_{1}+\tau}^{+\infty} \int_{v}^{+\infty} \int_{s}^{+\infty} \frac{h(u)}{\alpha(s) \beta(v)} d u d s d v \\
& \leq \leq \\
& \quad \frac{1}{c_{2}} \int_{t_{1}+\tau}^{t_{2}+\tau} \int_{v}^{+\infty} \int_{s}^{+\infty} \frac{h(u)}{\alpha(s) \beta(v)} d u d s d v+\frac{2 c_{1}}{c_{2}^{2}} \int_{T^{*}}^{+\infty} \int_{v}^{+\infty} \int_{s}^{+\infty} \frac{h(u)}{\alpha(s) \beta(v)} d u d s d v,
\end{aligned}
$$

which means that there exists $\delta>0$ such that

$$
\left|\left(S_{2 k} x\right)\left(t_{2}\right)-\left(S_{2 k} x\right)\left(t_{1}\right)\right|<\varepsilon, \quad x \in A(N, M), t_{1}, t_{2} \in\left[T, T^{*}\right] \text { with }\left|t_{2}-t_{1}\right|<\delta
$$

It is easy to verify that

$$
\left|\left(S_{2 k} x\right)\left(t_{2}\right)-\left(S_{2 k} x\right)\left(t_{1}\right)\right|=0<\varepsilon, \quad x \in A(N, M), t_{0} \leq t_{1} \leq t_{2} \leq T .
$$

That is, $S_{2 k}(A(N, M))$ is equicontinuous on $\left[t_{0},+\infty\right)$, and $S_{2 k}$ is completely continuous. By Lemma 1.2, there is $x_{0} \in A(N, M)$ such that $S_{1 k} x_{0}+S_{2 k} x_{0}=x_{0}$, which is a bounded nonoscillatory solution of (1.7).

Lastly, we demonstrate that (1.7) possesses uncountably many nonoscillatory solutions. Let $k_{1}, k_{2} \in\left(c_{1} N+\left(c_{1} / c_{2}\right) M, c_{2} M+\left(c_{2} / c_{1}\right) N\right)$ and $k_{1} \neq k_{2}$. For each $j \in\{1,2\}$, we choose $T_{j}>t_{0}$ and contraction mappings $S_{j k_{1}}$ and $S_{j k_{2}}$ satisfying (2.63)-(2.65) with $k$ and $T$ replaced by $k_{j}$ and $T_{j}$, respectively, and some $T_{3}>\max \left\{T_{1}, T_{2}\right\}$ satisfying

$$
\int_{T_{3}}^{+\infty} \int_{v}^{+\infty} \int_{s}^{+\infty} \frac{h(u)}{\alpha(s) \beta(v)} d u d s d v<\frac{c_{2}\left|k_{1}-k_{2}\right|}{2 c_{1}}
$$

Obviously, there are $x, y \in A(N, M)$ such that $S_{1 k_{1}} x+S_{2 k_{1}} x=x$ and $S_{1 k_{2}} y+S_{2 k_{2}} y=y$, respectively, and $x$ and $y$ are two bounded nonoscillatory solutions of (1.7) in $A(N, M)$. By (2.64) and (2.65), we gain that for $t \geq T_{3}$

$$
\begin{aligned}
x(t)= & \frac{k_{1}}{p(t+\tau)}-\frac{x(t+\tau)}{p(t+\tau)} \\
& +\frac{1}{p(t+\tau)} \int_{t+\tau}^{+\infty} \int_{v}^{+\infty} \int_{s}^{+\infty} \frac{1}{\alpha(s) \beta(v)} f\left(u, x\left(\sigma_{1}(u)\right), \ldots, x\left(\sigma_{n}(u)\right)\right) d u d s d v \\
y(t)= & \frac{k_{2}}{p(t+\tau)}-\frac{y(t+\tau)}{p(t+\tau)} \\
& +\frac{1}{p(t+\tau)} \int_{t+\tau}^{+\infty} \int_{v}^{+\infty} \int_{s}^{+\infty} \frac{1}{\alpha(s) \beta(v)} f\left(u, y\left(\sigma_{1}(u)\right), \ldots, y\left(\sigma_{n}(u)\right)\right) d u d s d v
\end{aligned}
$$


which together with (2.3), (2.61), and (2.62) mean that

$$
\begin{aligned}
|x(t)-y(t)| \geq & \frac{1}{p(t+\tau)}\left|k_{1}-k_{2}\right|-\frac{1}{p(t+\tau)}|x(t-\tau)-y(t-\tau)| \\
& -\frac{1}{p(t+\tau)} \int_{t+\tau}^{+\infty} \int_{v}^{+\infty} \int_{s}^{+\infty} \frac{1}{\alpha(s) \beta(v)} \\
& \times\left|f\left(u, x\left(\sigma_{1}(u)\right), \ldots, x\left(\sigma_{n}(u)\right)\right)-f\left(u, y\left(\sigma_{1}(u)\right), \ldots, y\left(\sigma_{n}(u)\right)\right)\right| d u d s d v \\
\geq & \frac{1}{c_{1}}\left|k_{1}-k_{2}\right|-\frac{1}{c_{2}}\|x-y\| \\
& -\frac{2}{c_{2}} \int_{T_{3}}^{+\infty} \int_{v}^{+\infty} \int_{s}^{+\infty} \frac{h(u)}{\alpha(s) \beta(v)} d u d s d v, \quad t \geq T_{3},
\end{aligned}
$$

which together with (2.75) yields that

$$
\|x-y\| \geq \frac{c_{2}}{1+c_{2}}\left(\frac{1}{c_{1}}\left|k_{1}-k_{2}\right|-\frac{2}{c_{2}} \int_{T_{3}}^{+\infty} \int_{v}^{+\infty} \int_{s}^{+\infty} \frac{h(u)}{\alpha(s) \beta(v)} d u d s d v\right)>0,
$$

that is, $x \neq y$. This completes the proof.

Theorem 2.7. Assume that there exist constants $M, N, c_{1}, c_{2}, T_{0}$ and a function $h \in C\left(\left[t_{0},+\infty\right), \mathbb{R}^{+}\right)$ satisfying (2.3), (2.4), and

$$
\begin{gathered}
0<\left(c_{1}-1\right) N<\left(c_{2}-1\right) M ; \\
-\infty<-c_{1} \leq p(t) \leq-c_{2}<-1, \quad t \geq T_{0} \geq t_{0}+\tau .
\end{gathered}
$$

Then (1.7) possesses uncountably many bounded nonoscillatory solutions in $A(N, M)$.

Proof. Set $k \in\left(\left(c_{1}-1\right) N,\left(c_{2}-1\right) M\right)$. It follows from (2.4) that there exists $T>T_{0}$ such that

$$
\int_{T}^{+\infty} \int_{v}^{+\infty} \int_{s}^{+\infty} \frac{h(u)}{\alpha(s) \beta(v)} d u d s d v<\min \left\{\left(c_{2}-1\right) M-k, \frac{c_{2}(k+N)}{c_{1}}-c_{2} N\right\}
$$

Define two mappings $S_{1 k}$ and $S_{2 k}: A(N, M) \rightarrow C\left(\left[t_{0},+\infty\right), \mathbb{R}\right)$ by

$$
\begin{gathered}
\left(S_{1 k} x\right)(t)= \begin{cases}\frac{-k}{p(t+\tau)}-\frac{x(t+\tau)}{p(t+\tau)}, & t \geq T, \\
\left(S_{1 k} x\right)(T), & t_{0} \leq t<T,\end{cases} \\
\left(S_{2 k} x\right)(t)= \begin{cases}\frac{1}{p(t+\tau)} \int_{t+\tau}^{+\infty} \int_{v}^{+\infty} \int_{s}^{+\infty} \frac{1}{\alpha(s) \beta(v)} f\left(u, x\left(\sigma_{1}(u)\right), \ldots, x\left(\sigma_{n}(u)\right)\right) d u d s d v, & t \geq T, \\
\left(S_{2 k} x\right)(T), & t_{0} \leq t<T\end{cases}
\end{gathered}
$$


for $x \in A(N, M)$. From (2.3), (2.80)-(2.82), we obtain that

$$
\begin{aligned}
& \left(S_{1 k} x\right)(t)+\left(S_{2 k} y\right)(t) \\
& \leq \frac{k}{c_{2}}+\frac{M}{c_{2}}+\frac{1}{c_{2}} \min \left\{\left(c_{2}-1\right) M-k, \frac{c_{2}(k+N)}{c_{1}}-c_{2} N\right\} \\
& \leq M, \quad x, y \in A(N, M), t \geq T, \\
& \left(S_{1 k} x\right)(t)+\left(S_{2 k} y\right)(t) \\
& \geq \frac{k}{c_{1}}+\frac{N}{c_{1}}-\frac{1}{c_{2}} \min \left\{\left(c_{2}-1\right) M-k, \frac{c_{2}(k+N)}{c_{1}}-c_{2} N\right\}, \\
& \geq N, \quad x, y \in A(N, M), t \geq T
\end{aligned}
$$

which implies that $S_{1 k}(A(N, M))+S_{2 k}(A(N, M)) \subseteq A(N, M)$. The rest of the proof is similar to the proof of Theorem 2.6 and is omitted. This completes the proof.

\section{Examples}

In this section we construct seven examples as applications of the results presented in Section 2.

Example 3.1. Consider the following third-order nonlinear neutral delay differential equation:

$$
\begin{gathered}
\left\{\frac{t^{3}}{e^{t}}\left[\frac{t}{\ln (1+t)}\left(x(t)+\frac{3 \sin \left(t^{2}-2\right)+1}{7} x(t-\tau)\right)^{\prime}\right]^{\prime}\right\}^{\prime} \\
+\frac{1}{e^{t}}\left(x^{2}\left(\frac{2 t^{2}}{1+3 t}\right)+x^{2}\left(t-\frac{1}{t}\right)\right)=0, \quad t \geq t_{0}
\end{gathered}
$$

where $\tau>0$ and $t_{0}>0$ are fixed. Let $n=2$, and let $M$ and $N$ be two positive constants with $M>7 N$ and

$$
\begin{aligned}
& \alpha(t)=\frac{t^{3}}{e^{t}}, \quad \beta(t)=\frac{t}{\ln (1+t)}, \quad \quad p(t)=\frac{3 \sin \left(t^{2}-2\right)+1}{7}, \\
& c_{1}=\frac{4}{7}, \quad c_{2}=\frac{2}{7}, \quad \sigma_{1}(t)=\frac{2 t^{2}}{1+3 t^{\prime}}, \quad \sigma_{2}(t)=t-\frac{1}{t}, \quad h(t)=\frac{2 M^{2}}{e^{t}}, \\
& f(t, u, v)=\frac{u^{2}+v^{2}}{e^{t}}, \quad(t, u, v) \in\left[t_{0},+\infty\right) \times \mathbb{R}^{2} .
\end{aligned}
$$

Obviously, (2.1)-(2.3) hold. On the other hand,

$$
\int_{t_{0}}^{+\infty} \int_{v}^{+\infty} \int_{s}^{+\infty} \frac{h(u)}{\alpha(s) \beta(v)} d u d s d v=M^{2} \int_{t_{0}}^{+\infty} \frac{\ln (1+v)}{v^{3}} d v<+\infty
$$


That is, (2.4) holds. Thus Theorem 2.1 means that (1.7) possesses uncountably many bounded nonoscillatory solutions in $A(N, M)$.

Example 3.2. Consider the following third-order nonlinear neutral delay differential equation:

$$
\left\{\frac{(2 t+1)^{3}}{t}\left[\left(4 t^{2}+4 t+5\right)\left(x(t)+\frac{2 t^{2}}{1+3 t^{2}} x(t-\tau)\right)^{\prime}\right]^{\prime}\right\}^{\prime}+\frac{2^{x(\sqrt{t}+1)}}{t^{2}}=0, \quad t \geq t_{0}
$$

where $\tau>0$ and $t_{0}>0$ are fixed. Let $n=1$, and let $M$ and $N$ be two positive constants with $\left(3 t_{0}^{2}+1\right) M>3\left(t_{0}^{2}+1\right) N$ and

$$
\begin{aligned}
& \alpha(t)=\frac{(2 t+1)^{3}}{t}, \quad \beta(t)=4 t^{2}+4 t+5, \quad p(t)=\frac{2 t^{2}}{1+3 t^{2}} \\
& c_{1}=\frac{2}{3}, \quad c_{2}=\frac{2 t_{0}^{2}}{1+3 t_{0}^{2}}, \quad \sigma_{1}(t)=\sqrt{t}+1, \quad h(t)=\frac{2^{M}}{t^{2}} \\
& f(t, u)=\frac{2^{u}}{t^{2}}, \quad(t, u) \in\left[t_{0},+\infty\right) \times \mathbb{R} .
\end{aligned}
$$

Obviously, (2.3), (2.25), and (2.26) hold. Moreover,

$$
\int_{t_{0}}^{+\infty} \int_{v}^{+\infty} \int_{s}^{+\infty} \frac{h(u)}{\alpha(s) \beta(v)} d u d s d v=\frac{2^{M}}{16}\left[\frac{1}{2\left(2 t_{0}+1\right)}-\frac{1}{2}\left(\frac{\pi}{2}-\arctan \left(2 t_{0}+1\right)\right)\right]<+\infty
$$

Hence (2.4) holds. Thus Theorem 2.2 ensures that (1.7) possesses uncountably many bounded nonoscillatory solutions in $A(N, M)$.

Example 3.3. Consider the following third-order nonlinear neutral delay differential equation:

$$
\left\{e^{t-t^{2}}\left[\left(t^{4}+1\right)^{1 / 3}\left(x(t)+\frac{-3 t-1}{5 t+2} x(t-\tau)\right)^{\prime}\right]^{\prime}\right\}^{\prime}+\frac{t x\left(t^{3}\right)}{e^{t^{2}}\left(1+x^{2}\left(t^{2}+2 t\right)\right)}=0, \quad t \geq 0
$$

where $\tau>0$ is fixed and $t_{0}=0$. Let $n=2$, and let $M$ and $N$ be two positive constants with $4 M>5 N$ and

$$
\begin{aligned}
& \alpha(t)=e^{t-t^{2}}, \quad \beta(t)=\left(t^{4}+1\right)^{1 / 3}, \quad p(t)=\frac{-3 t-1}{5 t+2}, \quad c_{1}=\frac{3}{5}, \\
& c_{2}=\frac{1}{2}, \quad \sigma_{1}(t)=t(t+2), \quad \sigma_{2}(t)=t^{3}, \quad h(t)=\frac{M t}{\left(1+N^{2}\right) e^{t^{2}}} \\
& f(t, u, v)=\frac{t v}{e^{t^{2}}\left(1+u^{2}\right)}, \quad(t, u, v) \in\left[t_{0},+\infty\right) \times \mathbb{R}^{2} .
\end{aligned}
$$


Clearly, (2.3), (2.25), and (2.30) hold, and

$$
\int_{t_{0}}^{+\infty} \int_{v}^{+\infty} \int_{s}^{+\infty} \frac{h(u)}{\alpha(s) \beta(v)} d u d s d v=\frac{M}{2\left(1+N^{2}\right)} \int_{t_{0}}^{+\infty} e^{-v}\left(t^{4}+1\right)^{-1 / 3} d v<+\infty
$$

hence (2.4) holds. Therefore Theorem 2.3 guarantees that (1.7) possesses uncountably many bounded nonoscillatory solutions in $A(N, M)$.

Example 3.4. Consider the following third-order nonlinear neutral delay differential equation:

$$
\left\{\frac{t^{3}+1}{t^{2} \arctan t}\left[\frac{1}{t}(x(t)+x(t-\tau))^{\prime}\right]^{\prime}\right\}^{\prime}+\frac{x^{2}\left(t^{2}-t\right)\left(x\left(t^{2}-t\right)+3\right)}{t^{4}}=0, \quad t \geq t_{0},
$$

where $\tau>0$ and $t_{0}>0$ are fixed. Let $n=1$, and let $M$ and $N$ be two positive constants with $M>N$ and

$$
\begin{array}{lll}
\alpha(t)=\frac{t^{3}+1}{t^{2} \arctan t^{\prime}}, & \beta(t)=\frac{1}{t}, p(t)=1, \quad \sigma_{1}(t)=t^{2}-t, \\
h(t)=\frac{M^{2}(M+3)}{t^{4}}, & f(t, u)=\frac{u^{2}(u+3)}{t^{4}}, & (t, u) \in\left[t_{0},+\infty\right) \times \mathbb{R} .
\end{array}
$$

Obviously, (2.3) holds. Furthermore,

$$
\int_{t_{0}}^{+\infty} \int_{v}^{+\infty} \int_{s}^{+\infty} \frac{h(u)}{\alpha(s) \beta(v)} d u d s d v=\frac{M^{2}(M+3)}{3} \int_{t_{0}}^{+\infty} \frac{v \arctan v}{v^{3}+1} d v<+\infty
$$

that is, (2.4) holds. Thus Theorem 2.4 ensures that (1.7) possesses uncountably many bounded nonoscillatory solutions in $A(N, M)$.

Example 3.5. Consider the following third-order nonlinear neutral delay differential equation:

$$
\left\{t^{3}\left[\frac{1}{t}(x(t)-x(t-\tau))^{\prime}\right]^{\prime}\right\}^{\prime}+\frac{x^{2}(t+(1 / t)+\ln (1+t))}{t^{3}}=0, \quad t \geq t_{0}
$$

where $\tau>0$ and $t_{0}>0$ are fixed. Let $n=1$, and let $M$ and $N$ be two positive constants with $M>N$ and

$$
\begin{gathered}
\alpha(t)=t^{3}, \quad \beta(t)=\frac{1}{t}, \quad p(t)=-1, \quad \sigma_{1}(t)=t+\frac{1}{t}+\ln (1+t), \\
h(t)=\frac{M^{2}}{t^{3}}, \quad f(t, u)=\frac{u^{2}}{t^{3}}, \quad(t, u) \in\left[t_{0},+\infty\right) \times \mathbb{R} .
\end{gathered}
$$


Obviously, (2.3) holds. Notice that

$$
\sum_{i=1}^{+\infty} \int_{t_{0}+i \tau}^{+\infty} \int_{v}^{+\infty} \int_{s}^{+\infty} \frac{h(u)}{\alpha(s) \beta(v)} d u d s d v=\frac{M^{2}}{16} \sum_{i=1}^{+\infty} \frac{1}{\left(t_{0}+i \tau\right)^{2}}<+\infty
$$

that is, (2.49) holds. Hence Theorem 2.5 implies that (1.7) possesses uncountably many bounded nonoscillatory solutions in $A(N, M)$.

Example 3.6. Consider the following third-order nonlinear neutral delay differential equation:

$$
\left\{\frac{t^{2}}{e^{t^{2}}}\left[\left(1+\ln ^{2} t\right)\left(x(t)+\left(\frac{5 \pi}{2}+\arctan t^{2 / 3}\right) x(t-\tau)\right)^{\prime}\right]^{\prime}\right\}^{\prime}+\frac{t}{e^{t^{2}}\left(x^{2}\left(t^{2}+1\right)+1\right)}=0, \quad t \geq t_{0},
$$

where $\tau>0$ and $t_{0}>0$ are fixed. Let $n=1$, and let $M$ and $N$ be two positive constants with $(5 / 2)(9 \pi-(5 / 2)) N<3((25 \pi / 4)-3) M$ and

$$
\begin{gathered}
\alpha(t)=\frac{t^{2}}{e^{t^{2}}}, \quad \beta(t)=1+\ln ^{2} t, \quad c_{1}=3 \pi, c_{2}=\frac{5 \pi}{2}, \\
p(t)=\frac{5 \pi}{2}+\arctan t^{2 / 3}, \quad \sigma_{1}(t)=t^{2}+1, \quad h(t)=\frac{t}{\left(N^{2}+1\right) e^{t^{2}}}, \\
f(t, u)=\frac{t}{\left(u^{2}+1\right) e^{t^{2}}}, \quad(t, u) \in\left[t_{0},+\infty\right) \times \mathbb{R} .
\end{gathered}
$$

Clearly, (2.3), (2.61), and (2.62) hold, and

$$
\int_{t_{0}}^{+\infty} \int_{v}^{+\infty} \int_{s}^{+\infty} \frac{h(u)}{\alpha(s) \beta(v)} d u d s d v=\frac{1}{2\left(N^{2}+1\right)}\left(\frac{\pi}{2}-\arctan \left(\ln t_{0}\right)\right)<+\infty
$$

that is, (2.4) holds also. Thus Theorem 2.6 ensures that (1.7) possesses uncountably many bounded nonoscillatory solutions in $A(N, M)$.

Example 3.7. Consider the following third-order nonlinear neutral delay differential equation

$$
\left\{\frac{1}{t}\left[\frac{t^{2}+1}{e^{t^{2}}}\left(x(t)+\left(\sin \left(t-\frac{1}{t}\right)+4 \cos (\ln t)-7\right) x(t-\tau)\right)^{\prime}\right]^{\prime}\right\}^{\prime}+\frac{t x^{2}\left(\sqrt{t^{2}+1}\right)}{e^{t^{2}}}=0, \quad t \geq t_{0}
$$


where $\tau>0$ and $t_{0}>0$ are fixed. Let $n=1$, and let $M$ and $N$ be two positive constants with $M>11 N$ and

$$
\begin{gathered}
\alpha(t)=\frac{1}{t}, \quad \beta(t)=\frac{t^{2}+1}{e^{t^{2}}}, \quad c_{1}=12, c_{2}=2, \\
p(t)=\sin \left(t-\frac{1}{t}\right)+4 \cos (\ln t)-7, \quad \sigma_{1}(t)=\sqrt{t^{2}+1}, \\
h(t)=\frac{t M^{2}}{e^{t^{2}}}, \quad f(t, u)=\frac{t u^{2}}{e^{t^{2}}}, \quad(t, u) \in\left[t_{0},+\infty\right) \times \mathbb{R} .
\end{gathered}
$$

Obviously, (2.3), (2.79), and (2.80) hold. Note that

$$
\int_{t_{0}}^{+\infty} \int_{v}^{+\infty} \int_{s}^{+\infty} \frac{h(u)}{\alpha(s) \beta(v)} d u d s d v=\frac{M^{2}}{4}\left(\frac{\pi}{2}-\arctan t_{0}\right)<+\infty,
$$

that is, (2.4) holds. It follows from Theorem 2.7 that (1.7) possesses uncountably many bounded nonoscillatory solutions in $A(N, M)$.

\section{References}

[1] J. Diblík and M. Růžičková, "Existence of positive solutions of a singular initial problem for a nonlinear system of differential equations," The Rocky Mountain Journal of Mathematics, vol. 34, no. 3, pp. 923-944, 2004.

[2] B. Dorociaková and R. Olach, "Existence of positive solutions of delay differential equations," Tatra Mountains Mathematical Publications, vol. 43, pp. 63-70, 2009.

[3] A. Elbert, "Oscillation/nonoscillation criteria for linear second order differential equations," Journal of Mathematical Analysis and Applications, vol. 226, no. 1, pp. 207-219, 1998.

[4] L. H. Erbe, Q. K. Kong, and B. G. Zhang, Oscillation Theory for Functional Differential Equations, Marcel Dekker, New York, NY, USA, 1995.

[5] C. Huang, "Oscillation and nonoscillation for second order linear differential equations," Journal of Mathematical Analysis and Applications, vol. 210, no. 2, pp. 712-723, 1997.

[6] N. J. Huang and H. X. Li, "Existence of nonoscillatory solutions for $n$th order nonlinear neutral functional-differential equations," Journal of Mathematical Analysis and Applications, vol. 216, no. 2, pp. 375-389, 1997.

[7] M. R. S. Kulenović and S. Hadžiomerspahić, "Existence of nonoscillatory solution of second order linear neutral delay equation," Journal of Mathematical Analysis and Applications, vol. 228, no. 2, pp. 436-448, 1998.

[8] Z. Liu and S. M. Kang, "Infinitely many nonoscillatory solutions for second order nonlinear neutral delay differential equations," Nonlinear Analysis, vol. 70, no. 12, pp. 4274-4293, 2009.

[9] H. Z. Qin, N. N. Shang, and Y. M. Lu, “A note on oscillation criteria of second order nonlinear neutral delay differential equations," Computers E Mathematics with Applications, vol. 56, no. 12, pp. 2987-2992, 2008.

[10] X. H. Tang and Y. Liu, "Bounded oscillation for second-order delay differential equations with unstable type in a critical case," Applied Mathematics Letters, vol. 16, no. 3, pp. 263-268, 2003.

[11] Q. G. Yang, L. J. Yang, and S. M. Zhu, "Interval criteria for oscillation of second-order nonlinear neutral differential equations," Computers \& Mathematics with Applications, vol. 46, no. 5-6, pp. 903918, 2003.

[12] Y. Zhou, "Existence for nonoscillatory solutions of second-order nonlinear differential equations," Journal of Mathematical Analysis and Applications, vol. 331, no. 1, pp. 91-96, 2007. 


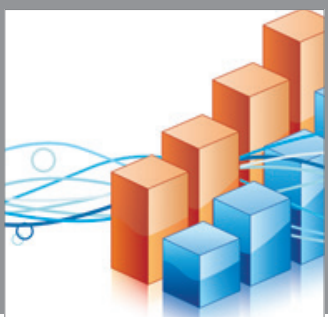

Advances in

Operations Research

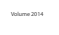

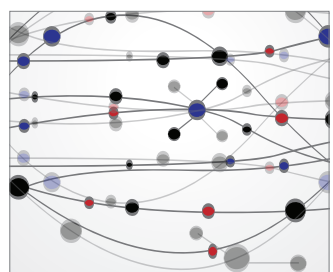

\section{The Scientific} World Journal
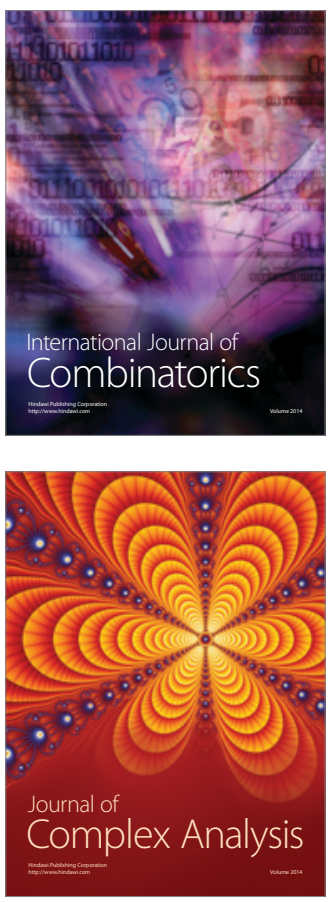

International Journal of

Mathematics and

Mathematical

Sciences
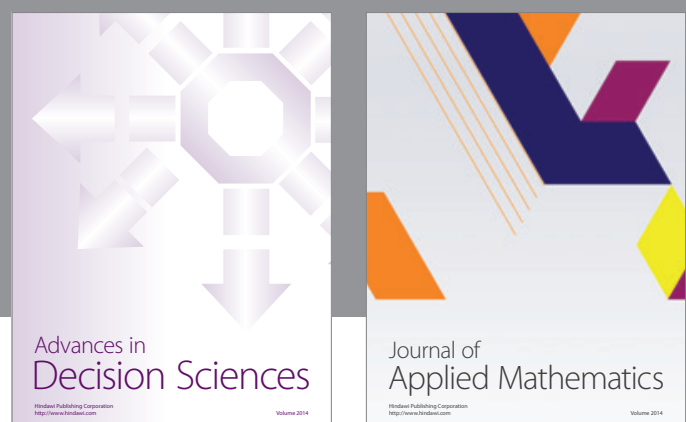

Journal of

Applied Mathematics
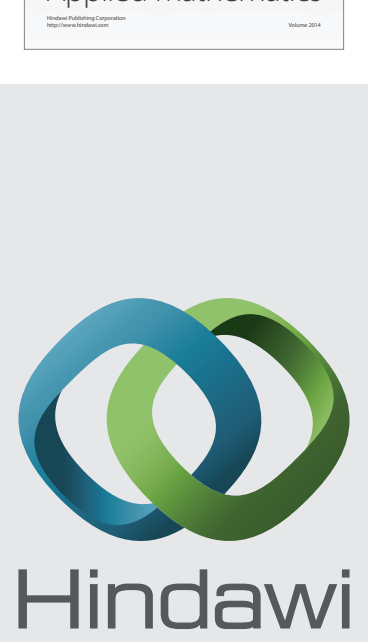

Submit your manuscripts at http://www.hindawi.com
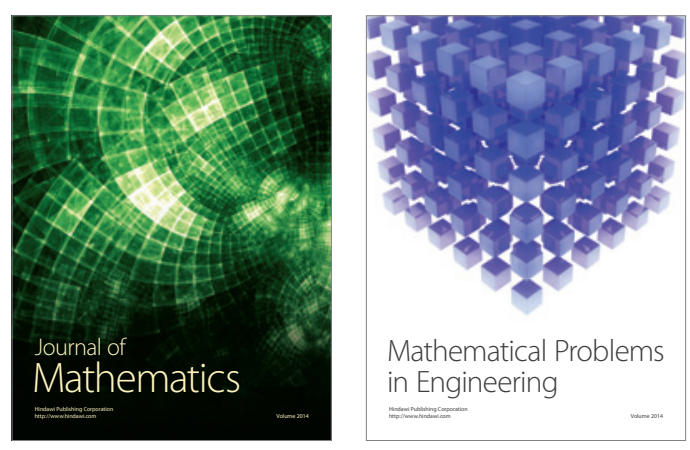

Mathematical Problems in Engineering
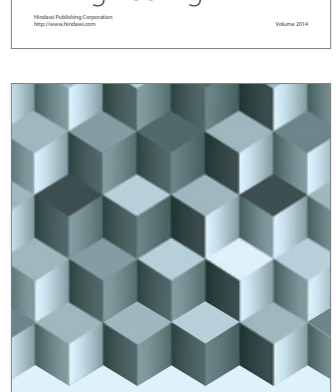

Journal of

Function Spaces
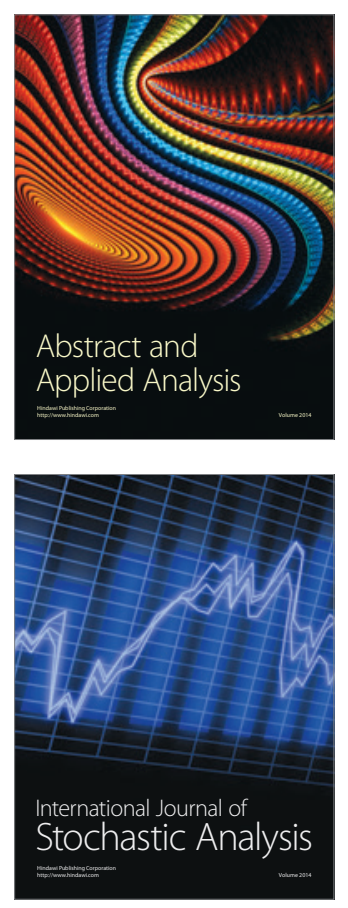

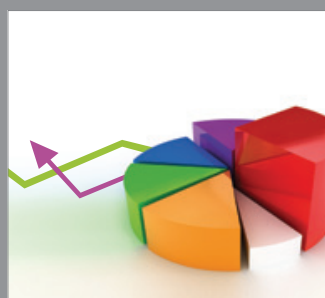

ournal of

Probability and Statistics

Promensencen
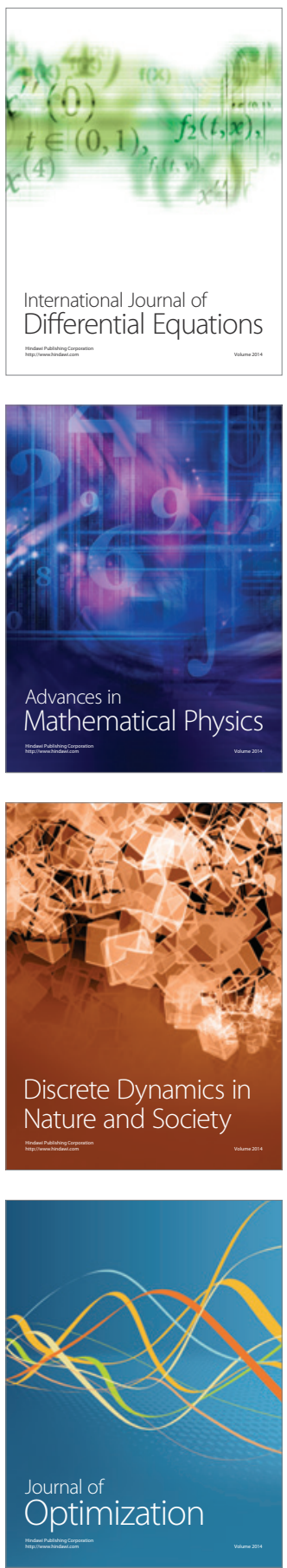